\title{
Effects of Anti-Smoking Advertising Messages on Smokers
}

\author{
Hayoung Park ${ }^{1}$, Cheolhan Lee ${ }^{2}$ \\ ${ }^{1}$ Ph.D. student, Dept. of Advertising \& PR, Dongguk University, South Korea, \\ christine.hayoung.park@gmail.com \\ ${ }^{2}$ Professor, Dept.of Advertising \& PR, Dongguk University, South Korea, clee@dongguk.edu \\ Corresponding Author: Cheolhan Lee
}

\begin{abstract}
This study analyzed the effects of anti-smoking advertising messages on smokers' attitudes toward tobacco. It is essential to understand the anti-smoking advertising because the campaign is conducted annually and mostly by the television media. The purpose of this study is to find the most effective message type among fear appeals, self-efficacy appeal, and social norm appeal for smokers. Only the smokers are included in this study, and their attitudes towards smoking were evaluated by experimental design. Thus, it is important to investigate on the effects of advertising messages such as fear appel, self-efficacy, and social norm toward the anti-smoking attitudes of the smokers. In addition, this study also examines the effects of the anti-smoking advertising messages on tabacco companies. In order to fulfill the purposes of this study, the researchers adopted experimental design and the ANOVA was adopted for the analysis. This study showed that the most effective advertising message was social norm message, followed by self-efficacy message. The fear appeal advertising message was least effective. In addition, the evaluations of tobacco companies were not influenced by the exposure of the anti-smoking advertising messages. This study also showed that intentions to quit smoking were not influenced by the advertising messages. However, the self-efficacy message was successful to change the smokers' attitude that smoking was harmful to society. Based on the findings, the researchers advised to adopt the self-efficacy ad message for the smokers because this message was the most effective for the smokers. It is also advised that anti-smoking ad itself does not affect negatively on tabacco companies. These results are intended to contribute to the future anti-smoking campaign designed for the smokers based on their message preference. Future studies are advised to include the popular endorsers, strength of fear appeals and specific anti-smoking techniques for the betterment of the persuasive effects for quitting tobacco.
\end{abstract}

Keywords: Anti-smoking Advertisement, Fear Appeal, Social Norm, Self-efficacy, Experimental Design, Smokers Participants

\section{Introduction}

According to the Korean National Cancer Center report, the tar and nicotine in the tobacco are the first degree cancer-causing agent and these agent leading to oral cancer, cancer of the larynx, cancer of the esophagus, cancer of stomach, and colorectal cancer[1]. Results of smoking harming the smokers and demand the social burdens in terms of national health insurance ranging from about 40 billion dollars in year 2005 to 60 billion dollars in year 2013. Approximately, the similar amount of the money were calculated for the loss of early death caused by smoking including medical expenditures and social care expenditures[2].

Received: April 23, 2021; $1^{\text {st }}$ Review Result: June 7, 2021; $2^{\text {nd }}$ Review Result: July 25, 2021 Accepted: August 30, 2021 
In order to lessen the detrimental effects of smoking by international health organizations, the South Korea Ministry of Health and Welfare is obliged to fulfill the FCTC(Framework on Tobacco Control). For the fulfilment, the increase of tobacco price, attachment of warning signs and pictures on the pack of tobacco product and the explications of detrimental elements causing cancers such as tar and nicotine. In addition, the smoking free zones should be set and fines should be imposed for the violators. Public media campaign featuring television ads with the help of national health promotion funds and setting of a national health promotion target to reduce cigarette smoking. A national quit line and nationwide ban on smoking in the restaurants and bars were also started. The nationwide smoking cessation medical services were started in the clinics and hospitals[3].

After signing the Framework Convention on Tobacco Control started by the World Health Organization in 2003, the Ministry of Health and Welfare of South Korea planned a more comprehensive anti-smoking campaign such as using mass media including television advertising, Internet advertising, outdoor advertising, media sponsorship on the various programs, and outdoor event promotions annually[4].

Among the media campaigns, the most expensive tool for anti-smoking ad is not fully examined as compared to its importance. Furthermore, the adult smokers are more or less neglecting the advertising messages of the effects on them. Thus, this study is aimed to further investigate on the adult smokers' responses on the various types of anti-smoking advertising messages based on the demographics of the study. If this study finds meaningful message effects on adults, the results are intended to contribute in producing more effective and persuasive messages. For these purposes, this study adopts the experiment for examining the effects of the ad messages based on the participation of the smokers.

\section{Content}

\subsection{Literature Review}

Despite the previous studies showed that anti-smoking advertising might play an important role in anti-smoking efforts, the effects are not always evident. This is because of some of the research results do not consider the fact that smokers have a tendency of selective exposure to the anti-smoking ad messages. In other words, smoking message in general has lower effects on smokers as compared to nonsmokers because smokers tend to reject the message. This study focused on the smokers only because it avoids the exaggerations of the effect that includes the non-smokers who already have negative attitudes toward tobacco[5].

It is true that not all the anti-smoking message are as effective as each other. Previous studies showed that fear appeal advertising message elicits strong emotional arousal because the messages more often than not include graphical portrayals of adverse results of the smoking. These graphical portrays due to the smoking related to cancers and other diseases might change the attitudes toward smoking from neutral to negative. However, for smokers, these tendency might not be applied because they evade the adverse advertisements based on fear appeals; thus, the advertising effects can be mitigated as compared with non-smokers[6]. Because of these mixed results, this study only recruited smokers and the fear message was exposed to them to find out the persuasive effects on them even though the participants tend to avoid the appeal.

In fact, South Korea has relied on the fear appeal in televised advertising campaign. In 2015, the antitobacco advertising theme was that "smoking is a disease". Apparently this campaign relied on fear appeal that smoking itself causes various disease such as cancer and heart attacks. For example, one milligram of tobacco can cause laryngeal cancer while having two packs of cigarettes can cause stroke. The visuals of these packages were shocking because of its visual effects and strong fear appeals. This approach is strong enough to provoke the smokers and they insisted that smokers are stigmatized by the 
Ministry of Health and Welfare[7].

However, the smokers resist the anti-tobacco messages because they protect their previous belief that smoking is okay for their health or they simply avoid the message that smoking causes many health problems and it might cause death eventually.

When the strong fear appeals ad message manifest its intention to threaten the smokers to quit, they said smokers negatively evaluate the message ads because they feel that they were attacked and their freedom to smoke were violated. This scenario is called psychological resistance[6].

However, such resistance does not always manifest. Some studies showed that fear appeal ad message is successfully executed and the perceived threat from the ad is closely related to anger, fear, and disgust towards smoking. The mixed findings are reported that fear leads to positive evaluation of the ad but also negatively related to the evaluations of attitudues toward smoking. In summary, it is almost unanimously reported that if the messages lead to the positive health effect among smokers by quitting chain smoking, the ad is regarded as more effective as compared to using other emotional appeals[7]. On the other hand, the social norm ad or social pressure message focuses on the social pressure of smoking. People who are emotion seekers tend to be more influenced by social norm ad message than people who are not. Thus, the social pressure ads seems to be more effective among people who are emotion seekers[8].

In other words, the ad message involving friends and coworkers' urge to quit smoking is effective when social bond is highly valued. The antismoking ads actually aired in Korea and the United States television showed that Korean smokers are more frequently exposed to social norm ads as compared to other types of message; however, self-efficacy ads are more frequently exposed in the U.S.[9].

Televised anti-tobacco smoking ads by the government and social institutions manifest their social responsibility to protect the health of the citizens. They use persuasive communication to solve social problems and elicit peoples' attitude towards seeking social goods[10].

Anti-smoking policies may influence the magnitude of socioeconomic differences in cigarette smoking as well as the overall smoking rates. Different policy measures can affect socioeconomic groups in different ways. With the enactment of the 1995 Health Promotion Act, the Korean government has developed numerous anti smoking policies including smoke-free buildings and zones, a public media campaign, and tobacco taxation.

Previous findings showed that there is a strong relationship between social norm ad message and intention to quit. In other words, the current research revealed that normative pressures for quitting cigarettes are effective as compared to other persuasive messages such as fear appeal, humor appeal, or self-efficacy appeal[5]. This result suggests that norm ad message might play a important role in how smokers processed the peers' pressure or social pressure in terms of quitting. The so-called resistance to message is well played against the acceptance of anti-smoking ad message.

Considering the accessibility of norm-based ad message belief, people will process the ad message differently because people with high sensitivity to peer groups may accept the social norm message more positively and will decide not to smoke. In other words, it is predicted that participants might process the ad message positively if the social norm based message precisely reaches its meaning that smoking hurts family, friends, or society. As long as the norm ad is made to more accessible, it might the increase of odds that smokers are more likely to quit smoking[7].

The researchers in this study anticipated that smokers are not easily influenced by fear appeal because they might resist the message as long as they smoke. Instead, social norm ad message is expected to be effective because the smokers process the social norm message more seriously than nonsmokers. It is also expected that social norm message is more accessible to smokers due to the fact that they had a good chance of being exposed to such pressures. However, self-efficacy ad is not popular and previous studies do not report the unanimous results in terms of message effect. Thus, self-efficacy ad message might be explored to further in this study. 
Based on the previous findings, this paper explores the effects of anti-tobacco ad messages and evaluates which message type is the most effective among smokers. The results of this study are expected to contribute to a better understandings of the effects of ad messages. For these purposes, this study provide four research questions as shown below.

\subsection{Research Questions}

Research question 1. Which among fear appeal, social norm, and self-efficacy anti-tobacco ad message type is the most effective?

Research question 2. How do smokers evaluate the tobacco company based on their anti-tobacco ad types?

Research question 3. How do smokers react to the quitting intention of the anti-tobacco ad types?

Research question 4. How do smokers react to the social attitudes of the anti-tobacco ad types toward smoking?

\subsection{Research Methods}

\subsubsection{Collection of Data}

This study recruited 390 smokers who were exposed to each type of anti-tobacco ad messages such as fear appeal message, social norm message, or self-efficacy message. The persuasive effects of antitobacco to the participants were examined in terms of cognition, attitude, and behavior. Also this study examined how the anti-tobacco ad messages affect the evaluations of the tobacco company.

\subsubsection{Stimulus Check}

The researchers recruited additional 30 participants to check the validity of stimulus. The 90 percent of participants exposed to fear appeal ad answered that they felt the severe threat to health. In addition, both participants exposed to social norm ad and self-efficacy ad answered they felt social pressure for smoking and gained self-confidence for non-smoking.

\subsubsection{Measurements}

The researchers measured the following ad reactions: the attitudes toward smoking, the attitudes toward tobacco companies, and the evaluation of the ad. The measurements in this study were adopted from the previous study in which the items were successfully confirmed[10]. The attitudes toward smoking was measured as the extent to which participants agreed or disagreed that smoking hurts other people's health, smoking causes various diseases, and smoking is not a desirable act for society. The attitudes toward the tobacco company was measured as the extent to which participants agreed or disagreed that the tobacco company is acceptable, the tobacco company is a likable company, and the tobacco company is attractive. The evaluation of the anti-tobacco ad was examined to the extent that the ad is likable, valuable, and interesting. All the results showed that the Cronbach alpha value were over .8 and valid.

\subsubsection{Descriptive statistics of participants}

This study recruited 390 participants and their demographic data are shown in [Table 1]. A total of 275 male smokers and 115 women smokers were recruited in this study. It is not easy to recruit women smokers because sometimes they do not reveal their smoking status. This study successfully recruited women smokers with the help of the professional research company. 
[Table 1] Gender and Age Descriptions of Participants

\begin{tabular}{|c|c|c|}
\hline & Number & Percent \\
\hline Gender & & \\
\hline Male & 275 & $70.5 \%$ \\
\hline Female & 115 & $29.5 \%$ \\
\hline Age & & \\
\hline $20-29$ & 148 & $37.9 \%$ \\
\hline $30-39$ & 120 & $30.7 \%$ \\
\hline $40-49$ & 122 & $31.3 \%$ \\
\hline
\end{tabular}

In terms of smokers' age, 20 to 29 year-old participants accounted for $38 \%$ of the sample followed by 40 to 49 year-old participants.

[Table 2] Income of the Participants

\begin{tabular}{|c|c|c|}
\hline & Number & Percent \\
\hline $\begin{array}{c}\text { Under 200 } \\
\text { (Ten Thousands Won) }\end{array}$ & 60 & $15.4 \%$ \\
\hline $200-300$ & 67 & $17.2 \%$ \\
\hline $300-400$ & 72 & $18.5 \%$ \\
\hline $400-500$ & 86 & $22.1 \%$ \\
\hline $500-600$ & 69 & $17.7 \%$ \\
\hline 600 over & 36 & $9.2 \%$ \\
\hline
\end{tabular}

As shown in [Table 2], the participants' income ranges from 200,000 or below to 600,000 and over. The most dominant income group answered $400-500$, which accounts for $22.1 \%$ of the sample. Only $9.2 \%$ consists of 600 or over. The variance of the income is well spread and their income does not affect the dependent variables.

As shown in [Table 3], the most dominant education group consists of undergraduates which accounts for $67.7 \%$ of the respondents. The professional undergraduate education group accounts for $15.9 \%$ while the graduate and over group accounts for $16.2 \%$. Only one high school dropout was reflected the collection.

[Table 3] Participants' Education Level

\begin{tabular}{|c|c|c|}
\hline & Number & Percent \\
\hline High school dropout and under & 1 & $.025 \%$ \\
\hline Professional undergraduate(2 years) & 62 & $15.9 \%$ \\
\hline Undergraduate (4 years) & 264 & $67.7 \%$ \\
\hline Graduate and over & 63 & $16.2 \%$ \\
\hline
\end{tabular}




\section{Results}

The first research question finds which among fear appeal, social norm, and self-efficacy message of anti-tobacco ads is the most effective. Based on the ANOVA test, the results showed that the self-efficacy ad message is statistically significantly. The mean score of social norm ad message is 3.77 . The selfefficacy ad message is also statistically significant. The mean score is 3.59. Meanwhile, no such effect found on fear appeal ad message. The results showed that it was not statistically significant to the smokers.

[Table 4] Anti-tobacco Ad Messages based on the Ad Types

\begin{tabular}{|c|c|c|c|c|}
\hline & Mean & MS & F & P \\
\hline Fear appeal message & 3.52 & .429 & 2.19 & .069 \\
\hline Social norm message & 3.59 & .752 & 3.905 & .004 \\
\hline Self- efficacy message & 3.77 & .584 & 3.005 & .018 \\
\hline
\end{tabular}

As shown on [Table 4], smokers do not assume that fear appeal message is as effective as social norm and self-efficacy message. In other words, fear appeal message is not persuasive among smokers. Thus, it is advised that future anti-tobacco messages should focus on either social norm message or selfefficacy message.

The second research question is about the evaluation of the tobacco company according to the antitobacco ad message type. The anti-tobacco ad messages might hurt the impressions on the tobacco companies because the messages more or less discuss the harmful effects of tobacco. However, no statistically significant result was found. Thus, anti-tobacco messages alone do not evoke the bad impressions toward the tobacco companies. It might come from the fact that tobacco companies put resources on the social campaigns to benefit the society, which negates the negative impressions of the anti-tobacco ad. For this matter, further research is needed to reveal the more precise effects.

[Table 5] Attitudes toward the Tobacco Companies based on the Types of the Message

\begin{tabular}{|c|c|c|c|c|}
\hline & Mean & MS & F & P \\
\hline Fear appeal message & 3.24 & 1.646 & 2.147 & .075 \\
\hline Social norm message & 3.25 & .401 & .523 & .719 \\
\hline Self- efficacy message & 3.13 & 1.297 & 1.692 & .164 \\
\hline
\end{tabular}

As shown on the [Table 5], the type of anti-tobacco ad message do not influence the evaluation of the tobacco company in a statistically significant way. In other words, no direct effect was found on the tobacco company's evaluation whether the anti-tobacco message was fear appeal, social norm appeal or self-efficacy appeal. It might come from the fact that the tobacco company put resources on social responsibility programs that affect smokers to evaluate the company more positively.

The third research question examines the smokers' intention to quit based on each anti-tobacco ad message. More specifically, this research question focuses on finding the difference of behavioral intentions to quit smoking among anti-tobacco ad messages. 
[Table 6] Intention to Quit Smoking according to Anti-tobacco Ad Messages

\begin{tabular}{|c|c|c|c|c|}
\hline & Mean & MS & F & P \\
\hline Fear appeal ad & 3.11 & .313 & .539 & .707 \\
\hline Social norm ad & 3.16 & .476 & .821 & .513 \\
\hline Self-efficacy ad & 3.23 & .726 & 1.521 & .289 \\
\hline
\end{tabular}

As shown on [Table 6], results showed that the ad message type do not influence the intention of quitting smoking among smokers. This means that all three ad messages failed to influence the behavioral level of smokers' reaction. Participants unanimously showed that they have the intention to quit smoking because they answered they wanted to quit this in the future, with the mean score of more than three points. However, no message effect was found at a statistically significant level. This result means at smokers are not decided to quit smoking based on one ad message. It implies that more complex decision making process exists for the smokers.

[Table 7] Attitude towards Social Cost for Smoking based on Each Message Type

\begin{tabular}{|c|c|c|c|c|}
\hline & Mean & MS & F & P \\
\hline Fear appeal message & 3.25 & 3.041 & 1.485 & .0544 \\
\hline Social norm message & 3.65 & .617 & 6.279 & .000 \\
\hline Self-efficacy message & 3.21 & .365 & .914 & .101 \\
\hline
\end{tabular}

Results showed that only social norm ad message has a statistically significant effect on accepting the social cost of smoking for the smokers. In other words, participants exposed to social norm ad showed that they answered smoking is a threat for society and can be an economic burden to society. In fact, fear appeal message was close for that effect; however, the result did not reach the .05 criteria. Self efficacy ad message do not have any effect.

As shown on [Table 7], only social norm ad message had a persuasive effect on social cost of tobacco smoking. Thus, if the government needs to highlight the social cost or health care expenditure for curing patients from smoking, it is advised to adopt the social norm ad message.

\section{Discussion and Conclusions}

\subsection{Discussion}

This study examined the anti-tobacco ad messages' effects on smokers, their evaluation of tobacco companies, and intention to quit. It is well documented that smokers' emotions influence their cessation behaviors. Antismoking ads are often designed to elicit certain emotional and motivation reactions in order to influence smokers to think about quitting and ultimately influence them to quit[11].

Emotions play an important role in smokers' decision to quit. Situations that cause smokers to experience negative emotions are usually related to smoking and negative emotion increases smokers' intention to smoke that predicts cessation failure. Previous studies on anti-tobacco campaign messages on cigarette warning labels found that graphic depictions of disease evoke strong negative emotions which increase smokers' thoughts about health risks, intentions to quit, and cessation behavior[12].

Graphic images about the negative consequences of smoking are designed to evoke feelings such as disgust, fear and ultimately, make people feel uncomfortable about smoking cigarettes. These antismoking messages challenge smokers' existing positive expectations about smoking and as a result, 
they experienced greater affective dissonance when dealing with inconsistent cognitions. Smokers who experience higher levels of affective dissonance are more likely to endorse beliefs about the addictive nature of smoking and report higher intentions to quit smoking[13].

Motivational factors also play an important role in smoking cessation. Making people feel motivated about quitting dominate the prediction of quitting trials. Anti-smoking ads that describe how to quit smoking elicited motivational reaction which was associated with quit attempt. The extent to which smokers perceive the antismoking message to be effective also influences their cessation behavior. Higher evaluations of perceived effectiveness of antismoking ads were associated with increased chance of smoking cessation attempts[14]. Perceived effectiveness, negative emotion, affective dissonance, and motivated reaction play important roles in smoking quitting behavior. These and the smokers' ad reactions were measured in this study.

Anti-smoking ads combine both message content and executional features such as graphic images in an effort to influence behavior change such as deterring tobacco purchase, making a quit attempt, or remaining quit. In this study, the researcher explore the following three ad types that represent commonly used anti-smoking advertising themes from the literature reviews. The fear appeal ad messages is closely related to negative health results focusing on the negative health consequences of smoking and include graphic images of health consequences[15]. Social norm ad messages means invoking meetings by emphasizing negative social results of smoking such as death from the secondhand smoking or increase of medicare expenses[16][17].

Self-efficacy ad message is closely related to the benefit of quitting and smokers are informed by the specific methods that provide detailed procedures of quitting. In general, tobacco is narcotic and it is not easy to quit by one's will only. Thus, self-efficacy ad message focused on the available resources that provide help from medical staff. In Korea, "no self trials, but helps by doctors make you quit smoking" is a typical way of boosting self-efficacy among smokers. It is also well known that young adults are heavily influenced by peers who urge them to smoke together. In that case, if the self-efficacy ad provides the ways to reject the peers' suggestion to smoke such as "say no to peers".

As shown above, the various practical approaches are discussed. The findings of this study could contribute in the future productions of anti-tobacco ads in terms of effective messages production. Even though it is apparently a barrier of smoker's resistance to the anti-tobacco message[18], social norm might be a good option[19]. In addition, more precisely targeted self-efficacy method can be another resource to rely on for smoking cessation campaign[20].

\subsection{Conclusions}

The purpose of this study is to examine the ad message effects and smokers' attitudes toward tobacco companies based on the influence of anti-tobacco ads. Based on findings, it was found that anti-tobacco ad messages have persuasive effects on attitudes toward smoking. Specifically, social norm message and self-efficacy message have persuasive effects on the evaluation of the advertising messages. Also, participants answered that social norm message contributed to realize that smoking leads to the increase of medical expenditure of the government and can be a threat to society. Such effects were not found in the attitudes toward tobacco companies and intention to quit.

One of the interesting finding of this study is that, fear appeal does not affect the smokers' evaluations on quitting. In general, no effect was found on the fear appeal that is contradictory to the previous studies. Partial explanation is that smokers do not easily change their attitude towards current smoking. It might be also from the fact the most studies recruited smokers and nonsmokers together; however, this study only focused on smokers.

Attitudes toward tobacco companies are not influenced by ad messages. Because of the active social responsibility programs such as giving, running sport teams, and youth education programs conducted 
by the tobacco companies, their evaluations were intact even though the anti-tobacco ad messages mentioned the harmful effects of the individual or social aspect. Further studies are needed to explore the evaluation of the tobacco companies.

To better understand the message type effects, future studies are advised to include the endorsement effects such as celebrities and humor appeal and consideration of the strength of fear appeals. These areas mentioned could contribute to the anti-tobacco campaign effects both academically and professionally.

\section{Acknowledgments}

This work was supported by the Dongguk University-Seoul Campus Sabbatical Research Fund of 2020.

\section{References}

[1] National Cancer Center, Korea current smoking reports based on statistics, (2016), https://www.cancer. go.kr/lay1S1T265C266/contents.do

[2] S. Lee, S. Lim, J. Pak, K. Han, H. Kang, Socioeconomic influence of major health threats and evaluations of regulation policy effects, National Health Insurance Policy Research Center, (2015), pp.691-707.

[3] M. Lee, S. Lim, S. Lee, H. Cho, Analyzing issues and a status invesigation of studies on tobacco control policies in South Korea, Health and Social Welfare Review, (2016), Vol.34, No.3, pp.165-191.

[4] Nonsmoking Guidance, http://www.nosmokinggudie.go.kr/index.do, December 31 (2017)

[5] M. Wakefield, B. Flay, M. Nicher, G. Giovino, Effects of anti-smoking advertising on youth smoking: A review, Journal of Health Communication, (2003), Vol.8, No.3, pp.229-247, DOI: 10.1080/10810730305686

[6] A. Kim, J. Nonnemaker, J. Guillory, P. Shafer, S. Parvanta, J. Holloway, .M. Farrelly, Antismoking ads at the point of sale: The influence of ad type and context on ad reactions, Journal of Health Communication, (2017), Vol.22, No.6, pp.477-487, DOI: 10.1080/10810730.2017.1311970

[7] S. A. Rains, The nature of Psychological Reactance Revisited: A meta-analytic review, Human Communication Research, (2013), Vol.39, No.1, pp.47-73, https://doi.org/10.1111/j.1468-2958.2012.01443.x

[8] Y. Kim, Y. Jang, An An Analysis of Influencing Factors on Smoking as a Risk Taking Behavior : With a Focus on Sensation Seeking, Social Smoking Conformity and Perceived Benefit/Barriers, Journal of Public Relations, (2015), Vol.19, No.2, pp.22-50, DOI : 10.15814/jpr.2015.19.2.22

[9] E. Hong, C. Lee, Content Analysis of Anti-Smoking TV advertisements: Different Adaptation of Health Communication Theories between Korea and the U.S.A., Journal of the Korea Contents Association, (2012), Vol.12, No.11, pp.76-87, https://doi.org/10.5392/JKCA.2012.12.11.076

[10] H. Jung, A study on semantics of smoking cessation TV public service advertisement, Journal of Cultural Product \& Desing, (2018), No.54, pp.77-87.

[11] J. Choi, G. Y. Noh, The effects of a stigmatizing anti-smoking campaign on autonomous vs. controlled motivation: The case of South Korea, Health Communication, (2020), Vol.35, No.9, pp.1073-1080.

[12] H. Soh, Exploring the response to the anti-smoking advertisements: Ad liking, empathy, and psychological resistance, The journal of the convergence on cultural technology, (2019), Vol.5, No.1, pp.99-105.

[13] E. L. Cohen, M. D. Shumate, A. Gold, Anti-smoking media campaign messages: theory and practice, Health Communication, (2007), Vol.22, No.2, pp.91-102, DOI: 10.1080/10410230701453884

[14] M. Wakefield, B. Flay, M. Nichter, G. Giovino, Effects of anti-smoking advertising on youth smoking: A review, 
Journal of Health Communication, (2003), Vol.8, No.3, pp.229-247.

[15] H. Cho, C. T. Salmon, Fear appeals of individuals in different stages of change: Intended and unintended effects and implications on public health campaign, Health Communication, (2006), Vol.20, No.1, pp.90-99.

[16] N. Rhodes, D. R. Roskos-Ewoldsen, A. Edison, M. B. Bradford, Attitudes and norm accessibility affect processing of anti-smoking messages, Health Psychology, (2008), Vol.27, No.3, pp.224-232.

[17] S. J. Kim, Reduction of the patient hygiene performance index based on an oral hygiene program using oral camera, International Journal of IT-based Public Health Management, (2020), Vol.7, No.1, pp.23-30.

[18] C. Kyung, H. Lee, S. Lim, Path model of mindfulness-based self-efficacy on rumination in mediation fields: Dual mediating effects of self-compassion and dysfunction attitudes, International Journal of Social Welfare Promotion and Management, (2020), Vol.7, No.2, pp.9-16.

[19] J. Geweke, D. L. Martin, Pitfails in drawing policy conclusions, from retrospective survey data: The case of advertising and underage smoking, Journal of Risk and Uncertainty, (2002), Vol.25, No.2, pp.111-131.

[20] P. B. Cialdini, Influence Science And Practice, $4^{\text {th }}$ ed, USA: Allyn \& Bacon Publishers, $(2000)$ 